\title{
Growth rate of early-stage hepatocellular carcinoma in patients with chronic liver disease
}

\author{
Chansik An', Youn Ah Choi ${ }^{2}$, Dongil Choi ${ }^{2}$, Yong Han Paik ${ }^{3}$, Sang Hoon Ahn', Myeong-Jin Kim', Seung Woon Paik ${ }^{3}$, \\ Kwang-Hyub Han ${ }^{4}$, and Mi-Suk Park ${ }^{1}$ \\ 'Department of Radiology, Research Institute of Radiological Science, Yonsei University College of Medicine, Seoul; ${ }^{2}$ Department of \\ Radiology and Center for Imaging Science, Samsung Medical Center, Sungkyunkwan University School of Medicine, Seoul; \\ ${ }^{3}$ Department of Medicine, Samsung Medical Center, Sungkyunkwan University School of Medicine, Seoul; ${ }^{4}$ Department of Medicine, \\ Yonsei University College of Medicine, Seoul, Korea
}

Background/Aims: The goal of this study was to estimate the growth rate of hepatocellular carcinoma $(\mathrm{HCC})$ and identify the host factors that significantly affect this rate.

Methods: Patients with early-stage HCC $(n=175)$ who underwent two or more serial dynamic imaging studies without any anticancer treatment at two tertiary care hospitals in Korea were identified. For each patient, the tumor volume doubling time (TVDT) of HCC was calculated by comparing tumor volumes between serial imaging studies. Clinical and laboratory data were obtained from the medical records of the patients.

Results: The median TVDT was 85.7 days, with a range of 11 to 851.2 days. Multiple linear regression revealed that the initial tumor diameter (a tumor factor) and the etiology of chronic liver disease (a host factor) were significantly associated with the TVDT. The TVDT was shorter when the initial tumor diameter was smaller, and was shorter in HCC related to hepatitis B virus (HBV) infection than in $\mathrm{HCC}$ related to hepatitis $\mathrm{C}$ virus (HCV) infection (median, 76.8 days vs. 137.2 days; $P=0.0234$ ).

Conclusions: The etiology of chronic liver disease is a host factor that may significantly affect the growth rate of earlystage HCC, since HBV-associated HCC grows faster than HCV-associated HCC. (Clin Mol Hepatol 2015;21:279-286)

Keywords: Hepatocellular carcinoma; Tumor burden; Follow-up studies; Liver neoplasm; Surveillance

\section{INTRODUCTION}

Hepatocellular carcinoma (HCC) is an important public health problem. It has an identifiable latent period, which necessitates an effective routine surveillance program.' Small HCCs detected by routine surveillance can often be cured with a good prognosis ( $>50 \%$ 5-year disease-free survival rate), whereas HCCs detected after the onset of symptoms have a poor prognosis ( $\leq 10 \% 5$-year survival rate). ${ }^{2}$ Furthermore, a randomized controlled study and a few uncontrolled studies have shown that HCC surveillance improves survival rates. ${ }^{3-5}$ Hence, HCC surveillance is widely used and recommended for at-risk populations. ${ }^{2,6,7}$

The surveillance process involves determining at-risk populations (who should enter the surveillance program), the surveillance (diagnostic) test, and the surveillance interval (how frequently the tests should be done). Although complete consensus has yet to be

\section{Corresponding author : Mi-Suk Park}

Department of Radiology, Severance Hospital, Yonsei University College of Medicine, 50 Yonsei-ro, Seodaemun-gu, Seoul 03722, Korea Tel: +82-2-2228-7400, Fax: +82-2-393-3035

E-mail:radpms@yuhs.ac
AASLD, the American Association for the Study of Liver Diseases; AFP, alpha-fetoprotein; ALT, alanine aminotransferase; APASL, the Asian Pacific Association for the Study of the Liver; EASL,the European Association for the Study of Liver; HBV, hepatitis B virus; HCC, hepatocellular carcinoma; HCV, hepatitis C virus; INR, International Normalized Ratio; PIVKA-II, protein induced by vitamin Kabsence 
reached on all three issues, the dilemmas faced by physicians caring for high-risk patients stem mainly from determining the optimal surveillance interval. ${ }^{8}$ The optimal surveillance interval is determined based on the tumor size and growth rate in order to detect HCCS at a stage when treatment is likely to produce a cure. ${ }^{9,10}$ While the ideal HCC surveillance interval is not known, intervals ranging from 3-12 months have been proposed. ${ }^{2,11,12}$ Currently, the American Association for the Study of Liver Diseases (AASLD), the European Association for the Study of Liver (EASL), and the Asian Pacific Association for the Study of the Liver (APASL) recommend that surveillance be conducted at 6-month intervals. ${ }^{2}$ This recommendation is based on the assumption that the HCC growth rate is similar in every patient. However, tumor growth in general is strongly affected by the microenvironment, ${ }^{13}$ and HCC doubling time is likely affected by host factors as well as by tumor factors.

Several previous studies have investigated HCC growth rates in order to determine the optimal surveillance or follow-up interval and reported several host factors that may affect the HCC growth rate, including sex, the doubling time of alpha-fetoprotein (AFP), and the presence of hepatitis $B$ surface antigen ( $\mathrm{HBs} A \mathrm{Ag})^{14-22}$ Those studies all had small sample sizes (fewer than 30 cases, even in the largest series), however, and reported a wide range of tumor volume doubling times (TVDTs) and inconsistent results for the significance of host factors. Therefore, we examined a relatively large number $(n=175)$ of untreated HCCs in patients with cirrhotic liver disease to obtain more solid estimates of TVDT and identify host factors that can significantly affect TVDT.

\section{PATIENTS AND METHODS}

\section{Study population}

This study was approved by the local institutional review boards of the two participating hospitals, and patient informed consent was waived. By searching the electronic database, we identified 15,890 patients diagnosed with HCC and underlying chronic liver disease between January 1, 2001 and December 31, 2010 at two tertiary care hospitals in Seoul, Korea. Two radiologists (C.A. and Y.A.C.) reviewed the medical records and imaging studies of each patient and identified 181 patients who met the following inclusion criteria: (1) untreated HCC detected by 3-phase or 4-phase dynamic CT or MRI with a slice thickness of $5 \mathrm{~mm}$ or less, (2) at least one follow-up 3-phase or 4-phase dynamic CT or MRI scan, (3) an interval of at least 1 month between the initial and follow- up studies without any anti-cancer treatment, and (4) met the Milan criteria (solitary HCC with a diameter $\leq 5 \mathrm{~cm}$ or up to $3 \mathrm{HCCs}$ with diameters $\leq 3 \mathrm{~cm}$ and no extrahepatic manifestation or vascular invasion). Six patients were excluded because they had either combined infection with hepatitis B virus (HBV) and hepatitis C virus (HCV) $(n=4)$ or a solitary HCC with a nodule in nodule appearance $(n=2)$. We defined HCC as either (1) a tumor $>1 \mathrm{~cm}$ in diameter with arterial enhancement and venous/delayed washout, (2) a hypervascular tumor $>1 \mathrm{~cm}$ in diameter that had grown either more than $5 \mathrm{~mm}$ or more than $50 \%$ in diameter since the previous exam, or (3) a tumor that was pathologically confirmed by biopsy within a month of initial detection. Our final study population comprised 175 patients with a combined total of 204 HCCs (122 men and 53 women; mean age, 60 years; age range, 21-85 years). Of these $204 \mathrm{HCCs}, 164$ (80.4\%) were defined as HCC because they were $>1 \mathrm{~cm}$ and showed the typical enhancement pattern (arterial enhancement and venous/delayed washout); $21(10.3 \%)$, hypervascular tumors $>1 \mathrm{~cm}$ that had grown; 9 $(4.4 \%)$, both showed the typical enhancement pattern and had grown; and 10 (4.9\%), confirmed by biopsy. These 175 patients did not receive treatment during the observation period because treatment was delayed $(n=92)$, treatment was refused $(n=36)$, the initial diagnosis was uncertain or incorrect $(n=20)$, the operative risk was high due to severe liver impairment or comorbidity $(n=19)$, or the patients were waiting for liver transplantation $(n=8)$. One hundred thirty-six of the patients eventually received treatment, which included loco-regional treatments such as transarterial chemoembolization or radiofrequency ablation $(n=101)$, hepatic resection $(n=20)$, liver transplantation $(n=10)$, and chemotherapy with or without local radiation therapy $(n=5)$. The remaining 39 patients did not receive treatment before loss to follow-up or death, primarily because they refused treatment or their physicians expected high morbidity or mortality related to the treatment.

\section{Data collection}

Electronic medical records were reviewed to obtain the clinical and laboratory data recorded for each patient within the first month before or after the initial imaging study. The clinical data included patient demographics, etiology of chronic liver disease, alcohol consumption, medication history, the presence of hepatic encephalopathy, and the type of treatment received. Alcohol intake was considered significant if it exceeded $25 \mathrm{~g}$ /day on average. ${ }^{23}$ Laboratory data included levels of alanine aminotransferase (ALT), AFP, protein induced by vitamin $\mathrm{K}$ absence (PIVKA-II), albu- 
min, bilirubin, international normalized ratio (INR), serologic markers of viral hepatitis, and HBV DNA. The reference values used for AFP and PIVKA-II levels were $<9 \mathrm{ng} / \mathrm{mL}$ and $<40 \mathrm{mAU} / \mathrm{mL}$, respectively. Chronic HBV infection was determined based on serum positivity for HBsAg for at least 6 months. Chronic HCV infection was determined based on serum positivity for both anti-HCV and HCV-RNA for at least 6 months. HBV DNA load was measured by the Digene hybrid capture assay (Digene Diagnostics, Beltsville, $M D$, USA), with a lower limit of $0.5 \mathrm{pg} / \mathrm{mL}$, or by the fully automated COBAS AmpliPrep-COBAS Taqman 96 system (Roche Molecular Systems, Branchburg, NJ, USA), with a linear detection range of $20-170,000,000 \mathrm{IU} / \mathrm{mL}$. The radiologists recorded the number and location of HCCS and the presence of ascites on the basis of CT and/or MRI scans. Child-Pugh score was calculated for each patient.

\section{Measurement of tumor volume doubling time}

This study included 3-phase and 4-phase contrast-enhanced dynamic $\mathrm{CT}$ and MRI studies. When more than two imaging studies were available, we selected two for TVDT calculation according to the following principles. First, two studies with the same imaging modality were chosen whenever possible. Second, if a patient had multiple follow-up examinations with the same imaging modality, only the follow-up with the maximum interval was used. Third, MRI was preferred over CT. The interval between the initial and last imaging studies ranged from 30 to 777 days (mean, 140 days; median, 91 days).

Two radiologists (M.S.P. and D.C.) who were unaware of the patients' clinical information measured the size of each HCC using the best available image of the tumor, with preference given to delayed images followed by portal phase images and finally arterial phase images. The average tumor diameter was calculated from the two greatest perpendicular dimensions, as measured on transverse images. The tumor size was then used to determine the tumor volume $(\mathrm{TV})$ calculated as TV $=(4 / 3) \times \varpi \times(\mathrm{D} / 2)^{3}$, where $D$ is the average diameter. On follow-up imaging, the same plane of section was used to measure the tumor size and volume. The growth rate of the HCC was expressed as TVDT calculated using Schwartz's equation ${ }^{24}:$ TVDT $=\left(T-T_{0}\right) \times \log 2 /\left(\log V / V_{0}\right)$, where $\left(T-T_{0}\right)$ indicates the time interval between two measurements, and $V_{0}$ and $V$ represent the tumor volumes at the two measurement times. Based on the calculated TVDT, the time needed for each $1 \mathrm{~cm}$ increase in tumor diameter was also calculated.

\section{Statistical analysis}

The TVDT was distributed lognormally. A Kolmogorov-Smirnov test revealed that the TVDT was normally distributed after log transformation $(P>0.15)$. Therefore, we used the log-transformed TVDT for statistical analysis. To analyze the TVDT among the patients, the HCC with the shortest doubling time was chosen for each patient that had multiple HCCs. Univariate and multivariate linear regression analyses were performed to find factors affecting the TVDT. The variables investigated were age, sex, alcohol consumption, antiviral medication, Child-Pugh class, virus type, serum AFP/PIVKA-II levels, AFP doubling time, initial average HCC diameter, and tumor multiplicity. Possible interactions of these variables with the different hospitals were tested in terms of their effects on TVDT. An ANOVA followed by Bonferroni post-hoc tests was performed to compare the TVDT among the different virus types. The relationship between the TVDT and the initial tumor diameter was estimated by a linear regression equation. Variables with alpha $<0.05$ in the univariate analysis were included in the multivariate analysis. The correlation between each factor in the multivariate analysis and the TVDT was analyzed using the Spearman rank correlation test. Two-sided $P$-values $<0.05$ were considered statistically significant. All statistical analyses were performed using SAS 9.2 (SAS Institute Inc., Cary, NC, USA).

\section{RESULTS}

\section{Baseline patient and tumor characteristics}

The patient and tumor characteristics are summarized in Table 1. Among the 175 patients, 116 (66.3\%) and 37 (21.1\%) were infected with HBV and HCV, respectively, and the remaining 22 $(12.6 \%)$ were not chronic carriers of hepatitis virus. Of the 161 patients who could be given Child-Pugh scores, $117(74.1 \%)$ were categorized as Child A, $26(16.5 \%)$ as Child B, and $15(9.5 \%)$ as Child $\mathrm{C}$. Among the patients for whom the data were available, 33.5\% (54/161) and $56.7 \%$ (55/97) had normal AFP and PIVKAII, respectively. We obtained the AFP doubling time for 132 patients, and 47 (35.6\%) of those had stable or decreasing AFP levels during the observation period. A total of 31 patients received antiviral medication during the observation period: 22 received lamivudine (Zeffix; GlaxoSmithKline, Greenford, UK); 4 received adefovir (Hepsera; Gilead Sciences Inc., Foster City, CA, USA); 3 received entecavir (Baraclude; Bristol-Myers Squibb, Princeton, NJ, USA); 1 received both lamivudine and adefovir; and 1 received a combination of pegylated interferon $\alpha$-2a (Pegasys; Roche, 
Table 1. Patient and tumor characteristics

\begin{tabular}{|c|c|}
\hline Variables & Patients $(n=175)$ \\
\hline Age (years), mean $\pm S D$ & $60 \pm 9.4$ \\
\hline Sex (male/female) & $126 / 53$ \\
\hline \multicolumn{2}{|l|}{ Alcohol consumption } \\
\hline None & $104(67.1)$ \\
\hline Occasional ( $\leq 25$ g/day) & $12(7.7)$ \\
\hline Significant (>25 g/day) & $39(25.2)$ \\
\hline NA & 20 \\
\hline Anti-viral medication & $31(17.7)$ \\
\hline \multicolumn{2}{|l|}{ Etiology of liver disease } \\
\hline HBV/HCV/non-viral & $\begin{array}{c}116(66.3) / 37(21.1) / \\
22(12.6)\end{array}$ \\
\hline \multicolumn{2}{|l|}{ Child-Pugh class } \\
\hline $\mathrm{A} / \mathrm{B} / \mathrm{C} / \mathrm{NA}$ & $\begin{array}{c}117(74.1) / 26(16.5) / \\
15(9.5) / 17\end{array}$ \\
\hline \multicolumn{2}{|l|}{ AFP (ng/mL) } \\
\hline$\leq 9$ & $54(33.5)$ \\
\hline$>9$ and $\leq 400$ & $93(57.8)$ \\
\hline$>400$ & $14(8.7)$ \\
\hline NA & 14 \\
\hline \multicolumn{2}{|l|}{ PIVKA-II (mAU/mL) } \\
\hline$\leq 40$ & $55(56.7)$ \\
\hline$>40$ and $\leq 400$ & $29(29.9)$ \\
\hline$>400$ & $13(13.4)$ \\
\hline NA & 78 \\
\hline \multicolumn{2}{|l|}{ Doubling time of AFP (days) } \\
\hline Stable or decreased & 47 (35.6) \\
\hline$>0$ and $\leq 150$ & $42(31.8)$ \\
\hline$>150$ & $43(32.6)$ \\
\hline NA & 43 \\
\hline Tumor multiplicity & $22(12.6)$ \\
\hline \multicolumn{2}{|l|}{ Initial tumor diameter (cm) } \\
\hline 1.5 & $94(53.7)$ \\
\hline$>1.5$ and 2 & $34(19.4)$ \\
\hline$>2$ and 3 & $40(22.9)$ \\
\hline$>3$ and 5 & $7(4)$ \\
\hline \multicolumn{2}{|l|}{ TVDT (days) } \\
\hline Median (range)/ geometric mean \pm SD & $85.7(11-851.2) / 91.4 \pm 2.2$ \\
\hline
\end{tabular}

Values are presented as median (range) or $\mathrm{n}(\%)$, unless stated otherwise. Percentages were calculated after excluding missing cases (if any).

$\mathrm{SD}$, standard deviation; NA, not assessable; HBV, hepatitis B virus; $H C V$, hepatitis C virus; AFP, alpha fetoprotein; PIVKA-II, protein induced by vitamin $\mathrm{K}$ absence; TVDT, tumor volume doubling time.
Seoul, Korea) and ribavirin (Viramid; Ilsung Pharmaceuticals, Seoul, Korea). Twenty-two patients (12.6\%) had more than 1 HCC: 15 had 2 HCCs, and 7 had 3 HCCs. The initial tumor diameter ranged from 1 to $4.6 \mathrm{~cm}$, with a median of $1.5 \mathrm{~cm}$; Of 175 HCCS, 94 (53.7\%) were $1.5 \mathrm{~cm}$ or smaller, 34 (19.4\%) were between $1.5 \mathrm{~cm}$ and $2 \mathrm{~cm}$, and the remaining 47 (26.9\%) were larger than $2 \mathrm{~cm}$. One hundred twenty-six HCCs (72\%) were found in the right hepatic lobe, while the other $49(28 \%)$ were found in the left or caudate lobe.

\section{Tumor volume doubling time and associated factors}

The TVDT ranged from 11 to 851.2 days (mean \pm SD, $127.6 \pm$ 128.7; median, 85.7 days), and its geometric mean \pm SD, computed by reverse-transforming the mean of the log-transformed values, was $91.4 \pm 2.2$. The univariate linear regression showed that a shorter TVDT was significantly associated with serum AFP $\geq$ $400 \mathrm{ng} / \mathrm{mL}$ (vs. $<9 \mathrm{ng} / \mathrm{mL}$ ), smaller initial tumor diameter, and HBV infection. In the multivariate analysis, virus type (HBV vs. $\mathrm{HCV}, P=0.0234)$ and initial tumor diameter $(P=0.0204)$ were independently associated with TVDT (Table 2). None of the variables investigated showed a significant interaction with hospital, so a subgroup analysis by hospital was not performed. The TVDT was shortest among the patients infected with HBV (median [range], 76.8 [11-752.2] days), longest among those infected with HCV (median [range], 137.2 [22.4-851.2] days), and intermediate among those with no hepatitis virus infection (median [range], 99.8 [33.6-288.4] days). There was a significant difference in

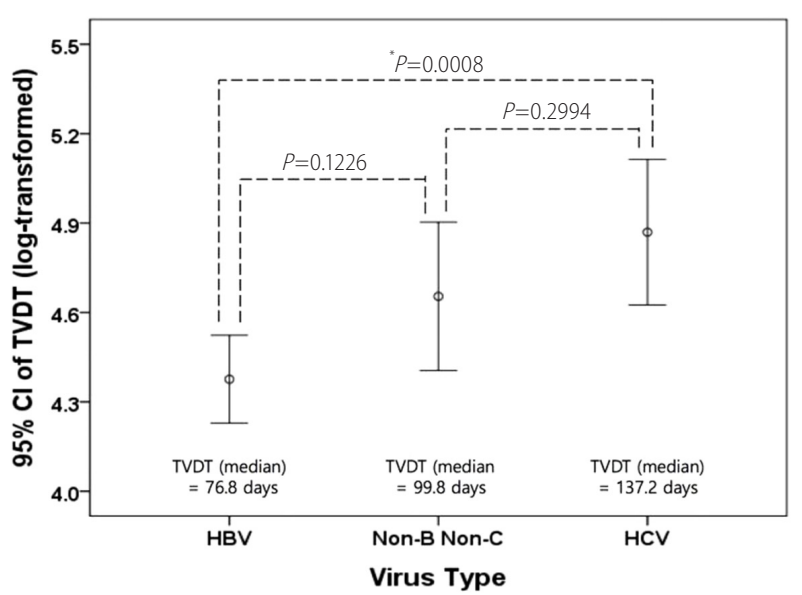

Figure 1. Difference in the TVDT according to virus type. HBV, HCV, and Non-B Non-C represent patients infected with HBV, infected with $\mathrm{HCV}$, and not infected with hepatitis virus, respectively. $\mathrm{Cl}$, confidence interval. "Statistically significant based on ANOVA followed by post-hoc Bonferroni analysis. TVDT, tumor volume doubling time; HBV, hepatitis B virus; $\mathrm{HCV}$, hepatitis $C$ virus. 
Table 2. Univariate and multivariate linear regression analyses to determine the factors related to the TVDT of untreated HCC

\begin{tabular}{|c|c|c|c|c|c|c|}
\hline & \multicolumn{3}{|c|}{ Univariate } & \multicolumn{3}{|c|}{ Multivariate } \\
\hline & Beta & SE & $P$-value & Beta & SE & $P$-value \\
\hline Age (years) ${ }^{*}$ & 0.005 & 0.006 & 0.4044 & & & \\
\hline \multicolumn{7}{|l|}{ Sex (vs. male) } \\
\hline Female & 0.023 & 0.131 & 0.8592 & & & \\
\hline \multicolumn{7}{|l|}{ Alcohol (vs. none) } \\
\hline Occasional ( $\leq 25 \mathrm{~g} /$ day) & -0.327 & 0.242 & 0.1779 & & & \\
\hline Significant (>25 g/day) & -0.072 & 0.145 & 0.6197 & & & \\
\hline Anti-viral medication & -0.054 & 0.147 & 0.7139 & & & \\
\hline Multiplicity of tumor & -0.063 & 0.178 & 0.7238 & & & \\
\hline \multicolumn{7}{|l|}{ Child-Pugh class (vs. A) } \\
\hline B & 0.204 & 0.177 & 0.2516 & & & \\
\hline C & -0.148 & 0.224 & 0.5102 & & & \\
\hline \multicolumn{7}{|l|}{ AFP (vs. $\leq 9)$} \\
\hline $9-400$ & -0.021 & 0.133 & 0.8770 & 0.030 & 0.134 & 0.8213 \\
\hline$\geq 400$ & -0.546 & 0.235 & 0.0216 & -0.458 & 0.232 & 0.0531 \\
\hline \multicolumn{7}{|l|}{ PIVKA-II (vs. $\leq 40)$} \\
\hline $40-400$ & 0.029 & 0.181 & 0.8724 & & & \\
\hline$\geq 400$ & -0.126 & 0.229 & 0.5836 & & & \\
\hline \multicolumn{7}{|c|}{ Doubling time of AFP (vs. <0) } \\
\hline $0-150$ & -0.305 & 0.158 & 0.0553 & & & \\
\hline$\geq 150$ & 0.144 & 0.156 & 0.3568 & & & \\
\hline \multicolumn{7}{|l|}{ Virus (vs. HBV) } \\
\hline None & 0.278 & 0.179 & 0.1226 & 0.256 & 0.192 & 0.1851 \\
\hline $\mathrm{HCV}$ & 0.493 & 0.144 & 0.0008 & 0.352 & 0.154 & 0.0234 \\
\hline Initial diameter ${ }^{*}$ & 0.174 & 0.078 & 0.0269 & 0.198 & 0.084 & 0.0204 \\
\hline
\end{tabular}

Variables with alpha $<0.05$ in the univariate analysis were included in the multivariate analysis.

*Beta was calculated per an increment of 1 year or $1 \mathrm{~cm}$.

TVDT, tumor volume doubling time; HCC, hepatocellular carcinoma; SE, standard error; AFP, alpha fetoprotein; PIVKA-II, protein induced by vitamin K absence; $\mathrm{HBV}$, hepatitis B virus; $\mathrm{HCV}$, hepatitis C virus.

TVDT between the patients infected with HBV and those infected with HCV $(P<0.001)$ but not between those with no hepatitis virus infection and those with either HBV or HCV infection (no virus vs. HBV, $P=0.1226$; no virus vs. $H C V, P=0.2994$; Fig. 1). There was a positive linear relationship between the log-transformed TVDT and the initial diameter of the HCC $\left(R^{2}=0.027\right.$; Fig. 2). The linear regression equations for the entire study cohort and the virus-type subgroups were as follows: for all patients, TVDT $=\exp$ $(0.173 \times$ diameter +4.211$)$; for patients with HBV, TVDT $=\exp$ $(0.124 \times$ diameter +4.161$)$; for patients with HCV, TVDT $=\exp$ $(0.221 \times$ diameter +4.475$)$; and for patients with no hepatitis virus, TVDT $=\exp (0.316 \times$ diameter +4.094$)$

We used the regression equations to calculate the time it would take an HCC to increase its diameter by $1 \mathrm{~cm}$ based on its initial diameter (from $1 \mathrm{~cm}$ to $5 \mathrm{~cm}$ ) and the virus type (HBV, HCV, or no virus) (Table 3). The estimated time needed for a HCC to grow from $1 \mathrm{~cm}$ to $2 \mathrm{~cm}$ was 212 days in patients with HBV infection and 328 days in those with HCV infection.

\section{DISCUSSION}

No prospective study has been performed to evaluate the HCC doubling time, and it is highly unlikely for ethical and practical reasons that one will ever be performed. The available evidence regarding the natural course of untreated HCC comes from retro- 
Table 3. Estimated time needed for a 1-cm increase in tumor diameter based on the initial tumor diameter and virus type

\begin{tabular}{llllc}
\hline \multirow{2}{*}{ Diameter change } & \multicolumn{4}{c}{ Estimated time (days) } \\
\cline { 2 - 5 } & HBV & NBNC & HCV & Overall \\
\hline $1 \mathrm{~cm} \rightarrow 2 \mathrm{~cm}$ & 212 & 296.9 & 328.4 & 240.5 \\
$2 \mathrm{~cm} \rightarrow 3 \mathrm{~cm}$ & 144.2 & 198 & 239.7 & 167.2 \\
$3 \mathrm{~cm} \rightarrow 4 \mathrm{~cm}$ & 115.8 & 192.7 & 212.5 & 141.1 \\
$4 \mathrm{~cm} \rightarrow 5 \mathrm{~cm}$ & 101.7 & 203 & 205.2 & 130.1 \\
\hline
\end{tabular}

HBV, hepatitis B virus; HCV, hepatitis C virus; NBNC, Non-B Non-C.

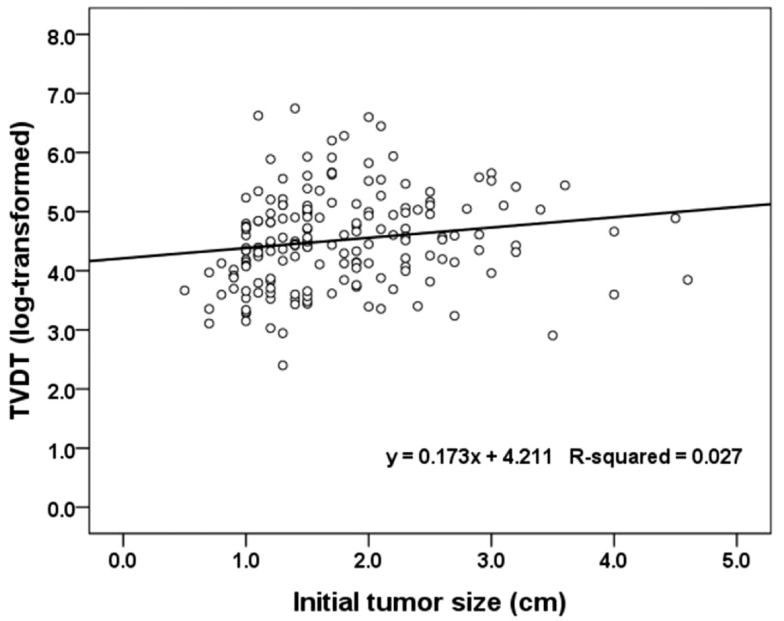

Figure 2. Scatter plot and linear regression line for the relationship between the initial tumor diameter and the TVDT of HCC. TVDT, tumor volume doubling time; $\mathrm{HCC}$, hepatocellular carcinoma.

spective studies. To our knowledge, our study is the largest series (175 patients) yet reported in the literature.

Our study shows that the growth rate of HCC may be significantly associated with the hepatitis virus type and the initial tumor diameter. Unlike the tumor diameter, the hepatitis virus type is a host factor, which suggests that the HCC growth rate may vary depending on the individual patient. In our study population, HCC grew faster in patients infected with HBV than in those infected with HCV. Although one previous study reported a shorter mean TVDT among HBsAg-positive patients (48 days for HBsAgpositive vs. 140 days for HBsAg-negative), ${ }^{14}$ other studies did not find a significant relationship between HCC growth rate and the presence of HBsAg. ${ }^{15,17,21}$ Each of the previous studies had relatively small numbers of patients overall and even smaller numbers in each subgroup (HBsAg positive and HBsAg negative). The resulting lack of statistical power in each study may have led to an increased probability of type II errors. In addition, most of the previous studies grouped patients with HCV infection and pa- tients with no hepatitis virus infection together as "HBsAg-negative patients" and therefore could have not evaluated a potential difference in HCC growth rate between patients infected with HBV and those infected with HCV. Our results suggest that their estimates of HCC growth rates in patients with HCV infection may have been higher if the previous studies grouped the patients with HCV infection separately from those with no hepatitis virus infection.

It is reported that the etiology of HCC affects the characteristics of HCC. ${ }^{25,26}$ Different HCC gene expression profiles are associated with different chronic liver disease etiologies, ${ }^{27-29}$ which may lead to different tumor behaviors in patients infected with HBV and $\mathrm{HCV}$. Furthermore, the morphologic and molecular characteristics of chronic viral liver disease vary significantly based on the virus type, and the resulting differences in the underlying liver parenchyma may impact on HCC growth through the interactions between cancer cells and the surrounding parenchyma. ${ }^{30,31}$ Although our finding revealed that HBV-associated HCC may grow faster than HCV-associated HCC, the underlying mechanism for this disparity remains uncertain. To our knowledge, no direct comparison was made between HBV- and HCV-transformed cells, regarding cell growth rate in vitro. ${ }^{31}$ It is of interest to define the molecular mechanism underlying the faster growth of HBV-associated HCC, but this is beyond the scope of this study.

In our study population, the TVDT of HCC increased with increasing tumor size, which is consistent with the results of previous studies. ${ }^{15,21}$ These results suggest that HCCs do not grow exponentially. Malignant tumors frequently undergo non-exponential growth, as the tumor growth pattern varies depending on the tumor characteristics and the environment. ${ }^{32} \mathrm{~A}$ sigmoidal growth model could explain an inverse relationship between the tumor growth rate and the tumor size, and several types of human cancer are reported to show such a growth pattern. ${ }^{33-35}$ One possible explanation for tumors following a sigmoidal growth pattern is that only the tumor cells near the tumor boundary have access to 
enough nutrients for growth. ${ }^{32}$

The growth rate of HCC could be intimately intertwined with the recall strategy for non-invasive HCC diagnosis. The AASLD and EASL guidelines both state that any new nodule less than 1 $\mathrm{cm}$ in diameter cannot be diagnosed as HCC and should be followed up until the nodule grows to the point at which it can be diagnosed by imaging criteria. They recommend follow-up every 4 months for the first year and every 6 months thereafter. ${ }^{9}$ In our study, the average time required for an HCC to grow from $1 \mathrm{~cm}$ to $2 \mathrm{~cm}$ in diameter was 212 days for patients with HBV infection and 328.4 days for patients with HCV infection. We suggest different follow-up intervals for indeterminate small $(<1 \mathrm{~cm})$ nodules detected on surveillance tests in patients infected with HBV or HCV.

Our study has a few shortcomings. First, our study population is biased because we only included patients for whom TVDT was obtainable. Second, most of the HCCs included in the study were diagnosed radiologically without biopsy. We strictly followed the AASLD Practice Guidelines for radiologic diagnosis, which have been prospectively validated by a study that reported high positive predictive values for such diagnoses (MRI alone, 97.4\%; combined MRI and contrast-enhanced ultrasonography, 100\%). ${ }^{2,36}$ Furthermore, we believe that a radiologic diagnosis better reflects real clinical situations, where treatment decisions for high-risk patients are made when HCC is suspected based on imaging studies. Third, TVDT was calculated on the assumption that a tumor is spherical in shape, although a tumor can have a variety of shapes.

In conclusion, we found that untreated early-stage HCC grew faster in patients infected with HBV than in those infected with HCV. This viral factor may be used as a reference when determining surveillance or recall policy for HCC.

\section{Acknowledgements}

This study was supported by a grant from the National Research Foundation of Korea (\#2012R1A1A3005720).

\section{Conflicts of Interest}

The authors have no conflicts to disclose.

\section{REFERENCES}

1. Daniele B, Bencivenga A, Megna AS, Tinessa V. Alpha-fetoprotein and ultrasonography screening for hepatocellular carcinoma. Gastroenterology 2004(5 Suppl 1);127:S108-112.
2. Bruix J, Sherman M; American Association for the Study of Liver Diseases. Management of hepatocellular carcinoma: an update. Hepatology 2011;53:1020-1022.

3. McMahon BJ, Bulkow L, Harpster A, Snowball M, Lanier A, Sacco $F$, et al. Screening for hepatocellular carcinoma in Alaska natives infected with chronic hepatitis B: a 16-year population-based study. Hepatology 2000;32:842-846.

4. Wong LL, Limm WM, Severino R, Wong LM. Improved survival with screening for hepatocellular carcinoma. Liver Transpl 2000;6:320325.

5. Zhang BH, Yang BH, Tang ZY. Randomized controlled trial of screening for hepatocellular carcinoma. J Cancer Res Clin Oncol 2004;130:417-422.

6. Kudo M. Japan's successful model of nationwide hepatocellular carcinoma surveillance highlighting the urgent need for global surveillance. Liver cancer 2012;1:141-143.

7. Kudo M. Early hepatocellular carcinoma: definition and diagnosis. Liver cancer 2013;2:69-72.

8. Teo EK, Fock KM. Hepatocellular carcinoma: an Asian perspective. Dig Dis 2001;19:263-268.

9. Della Corte C, Colombo M. Surveillance for hepatocellular carcinoma. Semin Oncol 2012;39:384-398.

10. Sherman M. Hepatocellular carcinoma: screening and staging. Clin Liver Dis 2011;15:323-334, vii-x.

11. Han KH, Kim do Y, Park JY, Ahn SH, Kim J, Kim SU, et al. Survival of hepatocellular carcinoma patients may be improved in surveillance interval not more than 6 months compared with more than 6 months: a 15-year prospective study. J Clin Gastroenterol 2013:47:538-544.

12. Kim DY, Han KH. Epidemiology and surveillance of hepatocellular carcinoma. Liver cancer 2012;1:2-14.

13. Kim Y, Stolarska MA, Othmer HG. The role of the microenvironment in tumor growth and invasion. Prog Biophys Mol Biol 2011;106:353379

14. Yoshino M. Growth kinetics of hepatocellular carcinoma. Jpn J Clin Oncol 1983;13:45-52.

15. Sheu JC, Sung JL, Chen DS, Yang PM, Lai MY, Lee CS, et al. Growth rate of asymptomatic hepatocellular carcinoma and its clinical implications. Gastroenterology 1985;89:259-266.

16. Ebara M, Ohto M, Shinagawa T, Sugiura N, Kimura K, Matsutani S, et al. Natural history of minute hepatocellular carcinoma smaller than three centimeters complicating cirrhosis. A study in $22 \mathrm{pa}$ tients. Gastroenterology 1986;90:289-298.

17. Okazaki N, Yoshino M, Yoshida T, Suzuki M, Moriyama N, Takayasu $K$, et al. Evaluation of the prognosis for small hepatocellular carcinoma based on tumor volume doubling time. A preliminary report. Cancer 1989;63:2207-2210.

18. Sadek AG, Mitchell DG, Siegelman ES, Outwater EK, Matteucci T, 
Hann HW. Early hepatocellular carcinoma that develops within macroregenerative nodules: growth rate depicted at serial MR imaging. Radiology 1995;195:753-756.

19. Ebara M, Hatano R, Fukuda H, Yoshikawa M, Sugiura N, Saisho $H$. Natural course of small hepatocellular carcinoma with underlying cirrhosis. A study of 30 patients. Hepatogastroenterology 1998;45(Suppl 3):1214-1220.

20. Kubota K, Ina H, Okada Y, Irie T. Growth rate of primary single hepatocellular carcinoma: determining optimal screening interval with contrast enhanced computed tomography. Dig Dis Sci 2003;48:581586.

21. Taouli B, Goh JS, Lu Y, Qayyum A, Yeh BM, Merriman RB, et al. Growth rate of hepatocellular carcinoma: evaluation with serial computed tomography or magnetic resonance imaging. J Comput Assist Tomogr 2005;29:425-429.

22. Park Y, Choi D, Lim HK, Rhim H, Kim YS, Kim SH, et al. Growth rate of new hepatocellular carcinoma after percutaneous radiofrequency ablation: evaluation with multiphase CT. AJR Am J Roentgenol 2008;191:215-220.

23. Corrao G, Bagnardi V, Zambon A, La Vecchia C. A meta-analysis of alcohol consumption and the risk of 15 diseases. Prev Med 2004;38:613-619.

24. Schwartz M. A biomathematical approach to clinical tumor growth. Cancer 1961;14:1272-1294.

25. Kim W, Oe Lim S, Kim JS, Ryu YH, Byeon JY, Kim HJ, et al. Comparison of proteome between hepatitis B virus- and hepatitis $C$ virusassociated hepatocellular carcinoma. Clin Cancer Res 2003;9:54935500.

26. Farazi PA, DePinho RA. Hepatocellular carcinoma pathogenesis: from genes to environment. Nat Rev Cancer 2006;6:674-687.

27. lizuka N, Oka M, Yamada Okabe H, Hamada K, Nakayama H, Mori N, et al. Molecular signature in three types of hepatocellular carcinoma with different viral origin by oligonucleotide microarray. Int J Oncol 2004;24:565-574

28. lizuka N, Oka M, Yamada-Okabe H, Mori N, Tamesa T, Okada T, et al. Comparison of gene expression profiles between hepatitis $B$ virus- and hepatitis C virus-infected hepatocellular carcinoma by oligonucleotide microarray data on the basis of a supervised learning method. Cancer Res 2002;62:3939-3944.

29. Lee C, Ling Z, Zhao T, Lee K. Distinct expression patterns in hepatitis $B$ virus- and hepatitis C virus-infected hepatocellular carcinoma. World J Gastroenterol 2008;14:6072-6077.

30. Kojiro M. Pathology of Hepatocellular Carcinoma. 1st edition ed, Wiley-Blackwell, 2006.

31. Arzumanyan A, Reis HM, Feitelson MA. Pathogenic mechanisms in HBV- and HCV-associated hepatocellular carcinoma. Nat Rev Cancer 2013;13:123-135.

32. Rodriguez-Brenes IA, Komarova NL, Wodarz D. Tumor growth dynamics: insights into evolutionary processes. Trends Ecol Evol 2013;28:597-604.

33. Guiot C, Degiorgis PG, Delsanto PP, Gabriele P, Deisboeck TS. Does tumor growth follow a "universal law"? J Theor Biol 2003;225:147151.

34. Reuss R, Ludwig J, Shirakashi R, Ehrhart F, Zimmermann H, Schneider $S$, et al. Intracellular delivery of carbohydrates into mammalian cells through swelling-activated pathways. J Membr Biol 2004;200:67-81.

35. Weedon Fekjaer H, Lindqvist BH, Vatten LJ, Aalen O, Tretli S. Breast cancer tumor growth estimated through mammography screening data. Breast Cancer Res 2008;10:R41.

36. Forner A, Vilana R, Ayuso C, Bianchi L, Solé M, Ayuso JR, et al. Diagnosis of hepatic nodules $20 \mathrm{~mm}$ or smaller in cirrhosis: Prospective validation of the noninvasive diagnostic criteria for hepatocellular carcinoma. Hepatology 2008;47:97-104. 